\title{
Combinatorial anti-angiogenic gene therapy in a human malignant mesothelioma model
}

\author{
SHUJI KUBO $^{1}$, MISATO TAKAGI-KIMURA ${ }^{1}$ and NORIYUKI KASAHARA ${ }^{2}$ \\ ${ }^{1}$ Department of Genetics, Hyogo College of Medicine, Nishinomiya, Hyogo, Japan; \\ ${ }^{2}$ Departments of Cell Biology and Pathology, University of Miami, Miami, FL, USA
}

Received April 29, 2015; Accepted June 2, 2015

DOI: $10.3892 /$ or.2015.4058

\begin{abstract}
Anti-angiogenic gene therapy represents a promising strategy for cancer; however, it has rarely been tested in malignant mesothelioma, a highly aggressive tumor associated with asbestos with poor prognosis. In the present study, we investigated whether anti-angiogenic factors such as angiostatin, endostatin and the soluble form of vascular endothelial growth factor receptor 2 (sFlk1) were able to inhibit endothelial cell proliferation via lentivirus-mediated gene transfer into malignant mesothelioma cells in culture. We also assessed whether a dual-agent strategy had greater therapeutic benefit. Human malignant pleural mesothelioma MSTO- $211 \mathrm{H}$ cells were transduced using lentiviral vectors that individually expressed angiostatin, endostatin and sFlk1 and linked to enhanced green fluorescent protein (EGFP) marker gene expression via an internal ribosome entry site. The lentivirus expressing EGFP alone was used as a control. The resultant cells designated as MSTO-A, MSTO-E, MSTO-F and MSTO-C were confirmed by western blot analysis and fluorescence microscopy to stably express the corresponding proteins. No differences were observed in the in vitro growth rates between any of these cells. However, co-culture of MSTO-A, MSTO-E and MSTO-F showed significant suppression of human umbilical endothelial cell growth in vitro compared with that of MSTO-C. Furthermore, a combination of any two among MSTO-A, MSTO-E and MSTO-F significantly enhanced efficacy. These results suggest that combinatorial
\end{abstract}

Correspondence to: Dr Shuji Kubo, Department of Genetics, Hyogo College of Medicine, 1-1 Mukogawa-cho, Nishinomiya, Hyogo 6638501, Japan

E-mail: s-kubo@hyo-med.ac.jp

Abbreviations: VEGF, vascular endothelial growth factor; EGFP, enhanced green fluorescent protein; HUVEC, human umbilical vein endothelial cells; sFlk1, soluble form of vascular endothelial growth factor receptor 2; LV-A, lentivirus vector expressing angiostatin; LV-E, lentivirus vector expressing endostatin; LV-F, lentivirus vector expressing sFlk1; LV-C, lentivirus vector expressing EGFP alone

Key words: anti-angiogenesis, angiostatin, endostatin, sFlk1, mesothelioma anti-angiogenic gene therapy targeting different pathways of endothelial growth factor signaling has the potential for greater therapeutic efficacy than that of a single-agent regimen.

\section{Introduction}

Malignant mesothelioma is a rare asbestos-induced aggressive cancer that arises from the mesothelial cells lining the pleural, peritoneal and pericardial cavities $(1,2)$. Conventional therapies for this malignancy include surgical resection, chemotherapy and irradiation; however, these measures are generally non-curative (2-4). Consequently, novel therapeutic paradigms are urgently required for the effective treatment of this aggressive and currently incurable malignancy.

Recent advances in understanding the regulation of physiological and pathological angiogenesis have revealed the processes by which tumors elicit an angiogenic response and have underscored their requirement for angiogenesis to sustain growth and metastasis (5). Proteins such as angiostatin (6-8) and endostatin (8,9) or vascular endothelial growth factor (VEGF) antagonists such as truncated forms of VEGF receptors (VEGFR) (10-12) have shown antitumor effects in preclinical cancer models. Preclinical studies have shown that angiogenesis has a key role in the biology of malignant mesothelioma $(13,14)$. Previous studies also have shown that mesothelioma cells highly express VEGF and its receptors, VEGFR-1/Flt-1 and VEGFR-2/Flk-1. VEGF stimulates mesothelioma cells growth in vitro in a dose-dependent manner and this growth has been shown to be inhibited by anti-VEGF antibodies (15). Mesothelioma patients have among the highest circulating VEGF levels of any solid tumor and high VEGF levels are a poor prognostic factor in this disease $(16,17)$. Hence, anti-angiogenic therapies could be effective in the treatment of patients with malignant mesothelioma.

Bevacizumab (Avastin ${ }^{\circledR}$ ), a VEGF-blocking monoclonal antibody, is widely used to treat metastatic colon and non-small cell lung cancer and ocular vascular proliferative disorders (18-20). Systemic administration of bevacizumab has been tested as a therapeutic for malignant pleural mesothelioma. Two non-randomized phase II trials of bevacizumab as maintenance strategy after six cycles of platinum-pemetrexed plus bevacizumab induction did not demonstrate an improvement in median progression-free survival compared with that of historical controls treated with pemetrexed/platinum 
combinations $(21,22)$. In a double-blind, placebo-controlled, randomized phase II trial, the addition of bevacizumab to gemcitabine-cisplatin followed by bevacizumab did not improve progression-free or overall survival rate in previously untreated patients with malignant pleural mesothelioma. These limited successes in clinical trials for mesothelioma are probably caused by the inability to achieve long-term and sustained therapeutic levels of anti-angiogenic protein at the tumor via systemic administration of bevacizumab, unlike the application of bevacizumab for the treatment of macular degeneration in which bevacizumab is injected directly into the eye (18). Therefore, persistent in situ production of anti-angiogenic factors via gene therapy would be an ideal strategy. We speculated that continuous production of anti-angiogenic factors at the tumors (in situ) by gene therapy would achieve maximum therapeutic effect of anti-angiogenic therapies.

In the present study, we investigated whether anti-angiogenic factors such as angiostatin, endostatin and the soluble form of VEGFR-2 (sFlk1) were able to inhibit endothelial cell proliferation via lentivirus-mediated gene transfer into MSTO-211H human malignant mesothelioma cells in culture. We also examined whether a dual-agent strategy could yield greater therapeutic benefit.

\section{Materials and methods}

Cell lines. Human malignant pleural mesothelioma MSTO-211H cells were obtained from American Type Culture Collection (ATCC, Manassas, VA, USA) and grown in Roswell Park Memorial Institute (RPMI) 1640 medium (Nacalai Tesque, Kyoto, Japan) supplemented with $10 \%$ heat-inactivated fetal calf serum (FCS; HyClone, Logan, UT, USA). Normal human umbilical vein endothelial cells (HUVEC) and their specific media, EGM-2, were purchased from Lonza Japan, Inc. (Tokyo, Japan). The cells were cultured in humidified $5 \% \mathrm{CO}_{2}$ at $37^{\circ} \mathrm{C}$.

Vector plasmids and virus production. Self-inactivating lentivirus vectors that individually expressed angiostatin (LV-A) and endostatin (LV-E) have been described previously as Sin-Ang (8) and Sin-End (8), respectively. The lentivirus vector, LV-F, was generated by cloning sFlk1 (provided by J. Folkman, Children's Hospital, Boston, MA, USA) into the Sin-GFP (8) vector construct. Each lentivirus vector contains an EGFP marker gene linked to the transgene expression cassette via an internal ribosome entry site. The lentivirus vector expressing EGFP alone (LV-C), which was described previously as Sin-Ang (8), was used as a control. The preparations of lentivirus vectors pseudotyped with vesicular stomatitis virus $G$ were produced by transient cotransfection of $293 \mathrm{~T}$ cells, as described previously $(23,24)$. The titers of these vectors were determined by fluorescent protein expression by using a FACSCalibur flow cytometer (Becton-Dickinson Japan, Tokyo, Japan) and expressed in terms of transducing units/milliliter.

Transduction of MSTO-211H mesothelioma cells with lentivirus vectors. MSTO-211H cells were cultured in growth medium to $30-50 \%$ confluency at the time of transduction $\left(1 \times 10^{6}\right.$ cells/well in 6 -well plates). Then, the cells were incubated with lentiviral vectors (LV-A, LV-E, LV-F or LV-C) at a multiplicity of infection of 10 for $72 \mathrm{~h}$; the cells were then subcultured three times to amplify by conventional culture methods and named MSTO-A, MSTO-E, MSTO-F cells and MSTO-C, respectively.

In vitro growth curves. The MSTO-C, MSTO-A, MSTO-E and MSTO-F cells were seeded in triplicate at $5 \times 10^{3} /$ well in 12-well culture plates. Cells in triplicate wells were harvested daily by trypsinization and the number of viable cells was determined by the trypan blue exclusion assay (Sigma-Aldrich Japan, Tokyo, Japan).

Sodium dodecyl sulfate-polyacrylamide gel electrophoresis (SDS-PAGE) and western blot analysis. To confirm the production and secretion of peptides after lentivirus infection, MSTO-C, MSTO-A, MSTO-E and MSTO-F cells were plated on two $10-\mathrm{cm}$ dishes at a density of $5 \times 10^{6} /$ dish. The next day, the media were replaced with $10 \mathrm{ml}$ of serum-free media and incubated for $48 \mathrm{~h}$. The conditioned media were collected and concentrated 20-fold on a Centricon YM-10 column (Merck-Millipore Japan, Tokyo, Japan); Ten microliters of each sample was subjected to SDS-PAGE using 5-20\% linear gradient gels (e-PAGEL; ATTO, Tokyo, Japan) and proteins were transferred to polyvinylidene fluoride membranes (Immobilon-P; Merck-Millipore Japan). For western blot analysis, rabbit anti-mouse angiostatin ab2904 (1:1000), rabbit anti-mouse endostatin ab58774 (1:250) or rabbit anti-human VEGF receptor 2 (Flk1) ab39638 (1:250) (Abcam, Cambridge, UK) were used as the primary antibodies and peroxidase-conjugated goat anti-rabbit antibody A6154 (1:2,000) (Sigma-Aldrich Japan) was used as the secondary antibody. Chemiluminescent detection of bound antibodies was performed by using the ECL system (ImmunoStar; Wako, Osaka, Japan).

Co-culture assay. MSTO-C, MSTO-A, MSTO-E and MSTO-F cells were harvested at near confluence using $0.05 \%$ trypsin/ EDTA solution and were subsequently counted. Then, $1 \times 10^{5}$ cells were seeded into Transwell chambers for 12-well culture plates with a $0.4 \mu \mathrm{m}$ pore-size (Corning Costar, Cambridge, MA). HUVEC cells $\left(5 \times 10^{3}\right)$ were plated on 12 -well plates with the appropriate culture medium. Following $24 \mathrm{~h}$ the transwell chambers were assembled on the 12-well plates for co-culture. After $70 \mathrm{~h}$, the cells were collected by trypsinization and counted.

To assess the combination effect of anti-angiogenic factors, MSTO-C,MSTO-A,MSTO-E and MSTO-F cells were harvested at near confluence; the cells were subsequently counted and mixed with each other at a ratio of $1: 1$ to create the following cell mixtures: MSTO-C/C, C/A, C/E, C/F, A/E, A/F and E/F. These mixed cells $\left(1 \times 10^{5}\right)$ were seeded in Transwell chambers. HUVEC cells $\left(5 \times 10^{3}\right)$ were plated on 12-well plates with the appropriate culture medium. The Transwell chambers were then assembled on the 12-well plates for co-culture $24 \mathrm{~h}$ later. After $70 \mathrm{~h}$, the cells were collected by trypsinization and counted.

Subcutaneous tumor models. MSTO-C, MSTO-A, MSTO-E and MSTO-F cells were harvested at near confluence using $0.05 \%$ trypsin/EDTA solution; the cells were subsequently counted and mixed with each other at a ratio of 1:1 to create the following cell mixtures: MSTO-C/C, C/A, C/E, A/E, $\mathrm{A} / \mathrm{F}$ and $\mathrm{E} / \mathrm{F}$. One million of the mixed cells in $100 \mu \mathrm{l}$ of $\mathrm{Ca}^{2+}$ - and $\mathrm{Mg}^{2+}$-free Hank's balanced salt solution were 

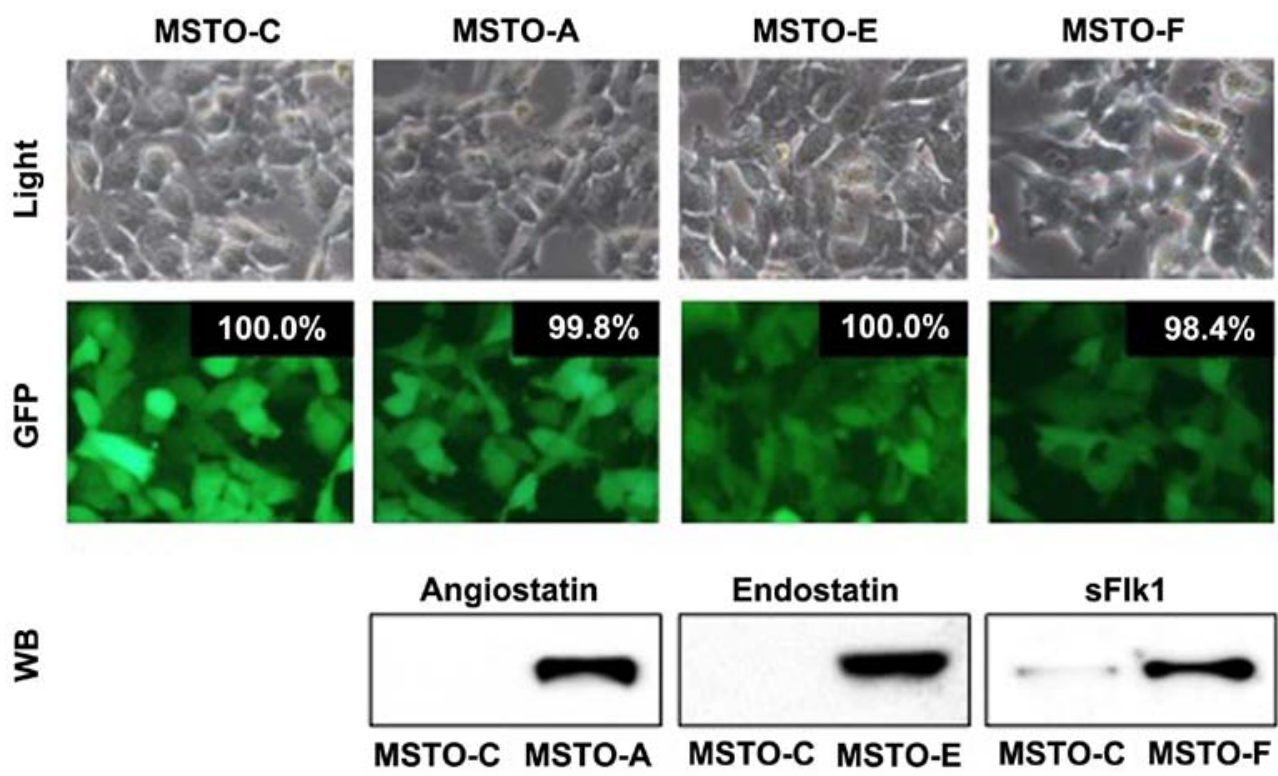

Figure 1. Transduction of human mesothelioma cells by lentivirus vectors expressing anti-angiogenic factors. Human malignant pleural mesothelioma MSTO-211H cells were transduced by using lentiviral vectors individually expressing angiostatin (LV-A), endostatin (LV-E) and soluble-Flk-1 (LV-F) and linked to EGFP marker gene expression via an internal ribosome entry site. The lentivirus expressing EGFP alone was used as a control (LV-C). The resultant cells, MSTO-A, MSTO-E, MSTO-F and MSTO-C, were analyzed for EGFP expression by fluorescence microscopy (\% of EGFP-positive cells are indicated as inset numbers). The conditioned media from the lentivirus vector-transduced cells were concentrated and the corresponding anti-angiogenic proteins were analyzed by western blot analysis (WB).

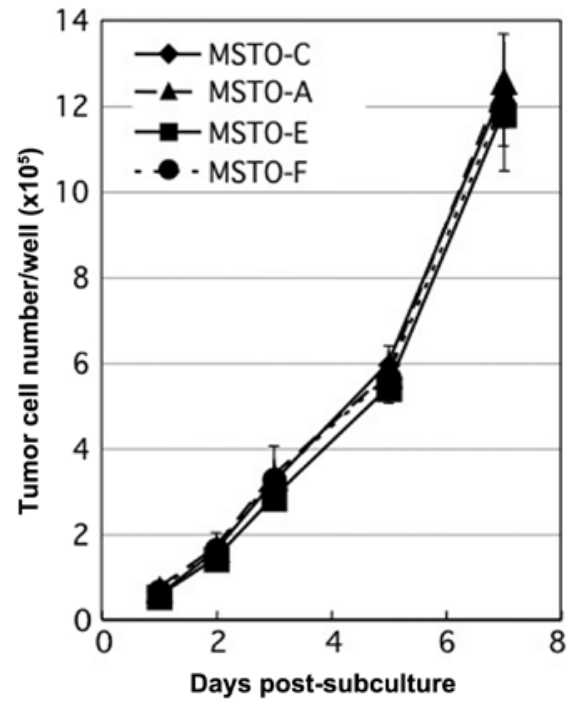

Figure 2. In vitro growth of MSTO-211H cells expressing anti-angiogenic factors. The growth rates at 7 days of the MSTO-A, MSTO-E, MSTO-F and MSTO-C cells were compared as determined by comparison of their proliferation in monolayer cultures. Data shown are the mean \pm SD calculated from triplicates.

injected subcutaneously on the dorsal flank of 6- to 7-weekold female BALB/c-nu/nu (nude) mice (Charles River Japan, Inc., Yokohama, Japan) (n=10/group). The mice were observed closely and tumors were measured by using a caliper every week over 12 weeks. Tumor volume was calculated as: $a \times b^{2} \times 0.5$, where $a$ and $b$ were the large and small diameters, respectively.

Statistical analysis. The results were presented as the mean \pm standard deviation (SD). The statistical significance of differences was calculated by using Student's t-test and a P-value of $<0.05$ was considered statistically significant.

\section{Results}

Transduction of human mesothelioma cells by lentivirus vectors expressing anti-angiogenic factors. Human malignant pleural mesothelioma MSTO-211H cells were transduced by using the lentiviral vectors LV-A, LV-E and LV-F and linked to EGFP marker gene expression via an internal ribosome entry site. LV-C was used as the control. As shown in Fig. 1, the resultant cells, MSTO-A, MSTO-E, MSTO-F and MSTO-C, were nearly 100\% EGFP-positive even after subculturing three times, which indicated highly efficient and stable transduction of MSTO$211 \mathrm{H}$ cells by the lentivirus vectors. The conditioned media from the lentivirus vector-transduced cells were concentrated and the corresponding anti-angiogenic proteins were analyzed by western blot analysis. In addition, all anti-angiogenic proteins were observed to be stably expressed and secreted into the cell culture medium after transduction by their respective vectors.

In vitro biological properties of MSTO-211H cells expressing anti-angiogenic factors. We investigated whether anti-angiogenic factors have any biological properties on MSTO-211 cells. Firstly, there was no difference in cell morphology among the MSTO-A, MSTO-E, MSTO-F and MSTO-C cells (Fig. 1). Secondly, no differences were observed in the in vitro growth rates after 7 days among the MSTO-A, MSTO-E, MSTO-F and MSTO-C cells ( $\mathrm{P}>0.05)$ (Fig. 2). These results showed that the expression and secretion of anti-angiogenic factors in MSTO-211H culture did not affect the morphology and growth of MSTO-211H by themselves in vitro.

Inhibition of HUVEC growth by co-culture with transduced MSTO-211H human mesothelioma cells expressing antiangiogenic factors. To investigate whether anti-angiogenic factors secreted from tumor cells inhibit the growth of 
A

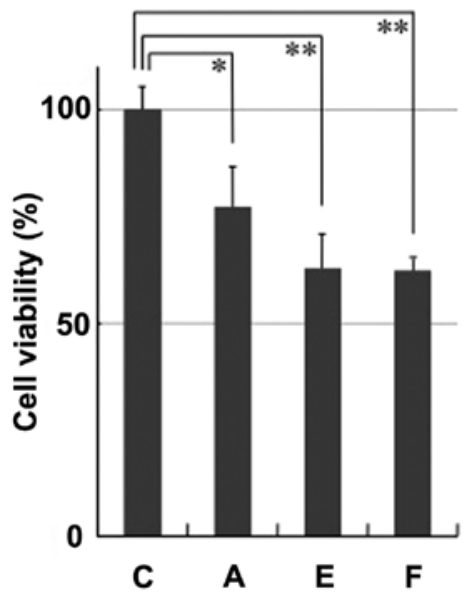

B

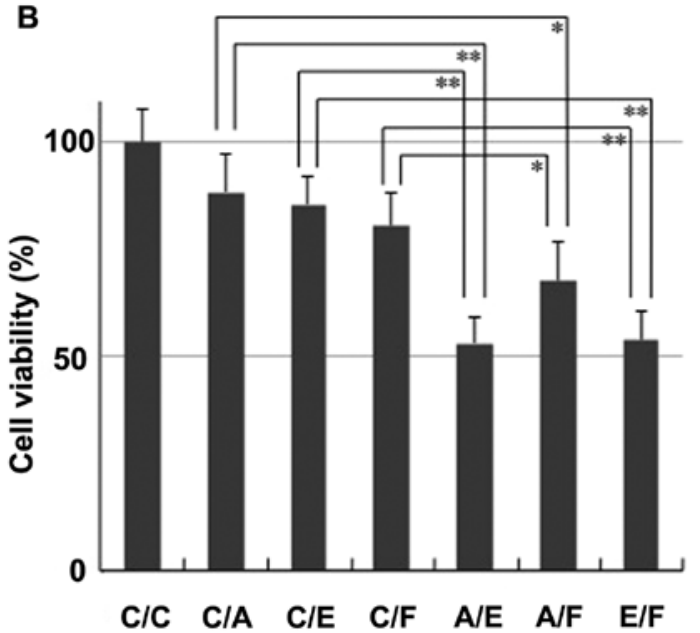

Figure 3. Inhibition of HUVEC proliferation by co-culture with transduced MSTO-211H human mesothelioma cells expressing anti-angiogenic factors. (A) Effect of a single anti-angiogenic factor on HUVEC growth. MSTO-C, MSTO-A, MSTO-E and MSTO-F cells on Transwell chambers were co-cultured with HUVECs on 12-well plates for $48 \mathrm{~h}$. HUVEC growth was then evaluated by cell count. Data were normalized to the value in MSTO-C without expressing any exogenous anti-angiogenic factors and shown as the mean $\pm \mathrm{SD}$ calculated from triplicates. ${ }^{*} \mathrm{P}<0.05$ and ${ }^{* *} \mathrm{P}<0.01$. (B) Combination effect of anti-angiogenic factors on HUVEC growth. MSTO-C, MSTO-A, MSTO-E and MSTO-F cells were harvested and mixed with each other at a ratio of 1:1 to create the following cell mixtures: MSTO-C/C, C/A, C/E, C/F, A/E, A/F and E/F. These mixed cells on Transwell chambers were then co-cultured with HUVECs on 12-well plates for $48 \mathrm{~h}$. HUVEC growth was then evaluated by cell count. Data were normalized to the value in $\mathrm{C} / \mathrm{C}$ without expressing any exogenous anti-angiogenic factors and shown as the mean \pm SD calculated from triplicates. ${ }^{*} \mathrm{P}<0.05$ and $^{* * *} \mathrm{P}<0.01$.

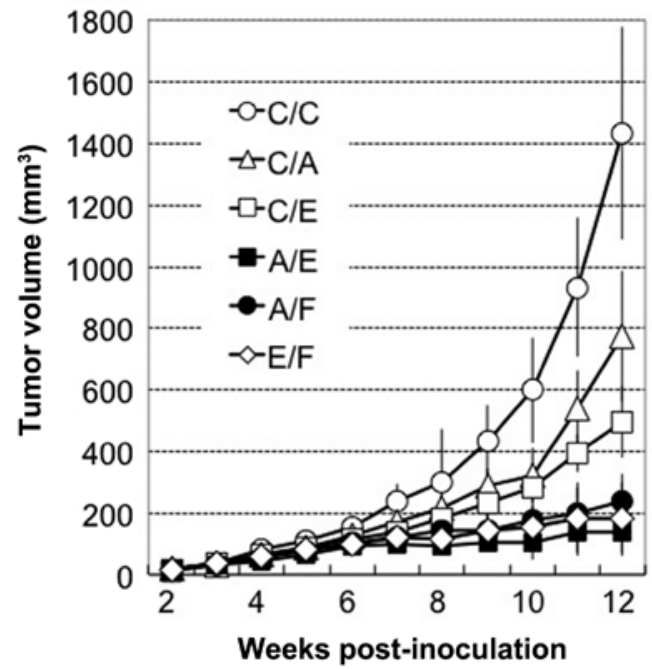

Figure 4. Combination effect of anti-angiogenic factors on subcutaneous mesothelioma tumor growth. MSTO-C, MSTO-A, MSTO-E and MSTO-F cells were mixed with each other at a ratio of $1: 1$ to create the following cell mixtures: MSTO-C/C, C/A, C/E, A/E, A/F and E/F. One million of the mixed cells were injected subcutaneously on the dorsal flank of nude mice ( $\mathrm{n}=10 /$ group). Tumor volumes were measured every week and data are shown are the mean $\pm \mathrm{SD}$.

adjacent endothelial cells, HUVEC cells were co-cultured with MSTO-C, MSTO-A, MSTO-E and MSTO-F cells in a dual-chamber assay (Fig. 3A). Co-culture of HUVEC cells with either MSTO-A (77.3 $\pm 11.6 \%, \mathrm{P}=0.0191)$, MSTO-E $(62.7 \pm 8.3 \%, \mathrm{P}=0.0009)$ or MSTO-F $(62.2 \pm 1.5 \%, \mathrm{P}=0.0002)$ cells resulted in significant inhibition of HUVEC cell proliferation compared with that of MSTO-C cells expressing no exogenous anti-angiogenic factors $(100.0 \pm 5.2 \%)$. These data demonstrate the ability of anti-angiogenic lentivirus vectors to mediate anti-proliferative effects on HUVEC cells indirectly via paracrine secretion from transduced cells.
For assessing the combination effect of anti-angiogenic factors secreted from tumor cells, HUVEC cells were cocultured with transduced cells mixed with MSTO-C, MSTO-A, MSTO-E and MSTO-F cells at a ratio of 1:1 in a dual-chamber assay (Fig. 3B). Inhibition effects on HUVEC cells in co-culture with any of the cells expressing single anti-angiogenic factors were MSTO-C/A $(88.5 \pm 8.8 \%, \mathrm{P}=0.1404), \mathrm{MSTO}-\mathrm{C} / \mathrm{E}$ (85.6 $\pm 6.3 \%, \mathrm{P}=0.0557)$ or $\mathrm{MSTO}-\mathrm{C} / \mathrm{F}(80.6 \pm 7.7 \%, \mathrm{P}=0.0266)$, and that in co-culture with cells expressing no exogenous anti-angiogenic factors was MSTO-C/C $(100.0 \pm 7.6 \%)$. These inhibition effects on HUVEC cells decreased (MSTO-C/A, $\mathrm{C} / \mathrm{E}$ and $\mathrm{C} / \mathrm{F}$ ) as compared with those in the previous experiment (MSTO-A, E and F) (Fig. 3A), probably because of a decrease in the percentages of MSTO-A, MSTO-E and MSTO-F cells by mixing with MSTO-C. Notably, any combination of anti-angiogenic factors resulted in enhanced inhibition (A/E, A/F and E/F; Fig. 3B), which suggested that the combinatorial anti-angiogenic therapy has the potential for greater therapeutic efficacy than that of a single-agent regimen.

In vivo combination effect of anti-angiogenic factors on subcutaneous mesothelioma tumor growth. To confirm the therapeutic advantage of combinatorial anti-angiogenic therapy, we examined in vivo antitumor efficacy in a subcutaneous MSTO xenograft model in athymic nude mice, which received subcutaneous injection of the following cell mixtures: MSTO-C/C, C/A, C/E, A/E, A/F and E/F. Tumors injected with the cells expressing single anti-angiogenic factors (C/A, $\mathrm{C} / \mathrm{E})$ resulted in a significant (46.1-65.4\%) reduction in tumor volume compared with that in the $\mathrm{C} / \mathrm{C}$ group $(\mathrm{P}<0.05)$ (Fig. 4). This reduction was unexpectedly high with respect to the in vitro co-culture data (Fig. 3B), which suggested that these anti-angiogenic factors possess an indirect effect in vivo. Furthermore, the combination of any two among MSTO-A, MSTO-E and MSTO-F significantly enhanced the antitumor 
efficacy compared with that of the single anti-angiogenic factor group (MSTO-C/A and C/F), which indicated the feasibility of the combinatorial anti-angiogenic therapy in vivo.

\section{Discussion}

Tumors elicit an angiogenic response and have been shown to require angiogenesis to sustain growth and metastasis (5). For example, VEGF is produced by a variety of tumors, including malignant mesothelioma and stimulate neovascularization of tumors $(4,25)$. Endothelial cells engaged in angiogenesis express VEGFR-1 and VEGFR-2; however, they produce only low levels of endogenous VEGF for an autocrine/paracrine signal required for endothelial cell survival and vascular homeostasis mainly through intracellular VEGFR-2 $(26,27)$. In addition, VEGFR-2 appears to play a more important role in initiating signal transduction pathways within endothelial cells because of its greater kinase activity and thus, in activating multiple signaling networks that lead to increased proliferation, sprouting, migration and tube formation of endothelial cells (28). Similar to the case described above, anti-angiogenic factors may function as an autocrine or paracrine growth factor for endothelial cells or several types of tumor cells, including mesothelioma. Therefore, continuous in situ production of anti-angiogenic factors at the tumors by gene therapy would achieve maximum therapeutic effect of anti-angiogenic therapies. As expected, in the present study, even a single factor significantly suppressed vascular endothelial growth. However, the anti-angiogenic factors, angiostatin, endostatin and sFlk1, did not affect the morphology and growth of any MSTO-211H cells producing anti-angiogenic factors (Fig. 1 and 2). This observation is partially consistent with a previous observation that MSTO-211H expressed VEGFR-2 at the mRNA level but did not produce detectable VEGFR-2 at the protein level (29). Therefore, other growth factor receptors and their signaling pathways may be much more important for the proliferation of this cell line.

In addition, we also showed that a dual-agent strategy may yield greater therapeutic benefit. The anti-angiogenic activities of angiostatin and endostatin interfere with the $\alpha_{\mathrm{V}} \beta_{3}$ integrin-mediated signaling in endothelial cells (6-9), although sFlk1 uses a different pathway through VEGF-signaling blockage (10-12). Because the combination of angiostatin and endostatin that have the same mechanism of anti-angiogenic action could yield greater therapeutic benefit than a single factor, the integrin pathway may not be saturated even by continuous in situ production of a single factor. Our data suggest that in situ continuous production of combined anti-angiogenic factors would be absolutely required at levels that cannot be achieved by oral or intermittent systemic administration. However, antiangiogenic gene therapy targeting the different pathways of endothelial growth factor signaling should be better for longterm success by presumably reducing the risk of resistance.

Anti-angiogenic therapy cannot eradicate tumors by itself and therefore, is a kind of second-line maintenance therapy. Therefore, long-term and combination treatment regimens will be essential in a clinical setting. In the present study, we used lentivirus-based vectors that allowed permanent integration and hence, the potential for stable expression of the delivered transgene. Alternatively, retroviral replicating vectors (RRVs) would be feasible in combination with RRV-mediated prodrug activator gene therapy against malignant mesothelioma because they can keep producing anti-angiogenic factors while a tumor exists $(30,31)$. As a combination, oncolytic viruses such as adenovirus and measles that can selectively kill tumor cells would be feasible because they can keep producing anti-angiogenic factors while viruses exist in the mesothelioma tumors (32-34).

In conclusion, continuous in situ production of anti-angiogenic factors at tumors by combinatorial anti-angiogenic gene therapy targeting the same and/or different pathways of endothelial growth factor signaling has the potential for greater therapeutic efficacy than that of a single-agent regimen.

\section{Acknowledgements}

We thank Atsuko Tamamoto, Nobutaka Okamura and members of the Joint-Use Research Facilities of the Hyogo College of Medicine for their technical assistance; Toshiaki Shichinohe for a part of the lentiviral constructs and Judah Folkman for sFlk1 plasmid. The present study was supported by a Grant-in-Aid for Researchers, Hyogo College of Medicine and a Grant-in-Aid for Scientific Research from the Ministry of Education, Culture, Sports, Science and Technology of Japan (25460484).

\section{References}

1. Ismail-Khan R, Robinson LA, Williams CC Jr, Garrett CR, Bepler G and Simon GR: Malignant pleural mesothelioma: A comprehensive review. Cancer Control 13: 255-263, 2006.

2. Tsao AS, Wistuba I, Roth JA and Kindler HL: Malignant pleural mesothelioma. J Clin Oncol 27: 2081-2090, 2009.

3. van der Most RG, Robinson BW and Nelson DJ: Gene therapy for malignant mesothelioma: Beyond the infant years. Cancer Gene Ther 13: 897-904, 2006.

4. Kotova S, Wong RM and Cameron RB: New and emerging therapeutic options for malignant pleural mesothelioma: Review of early clinical trials. Cancer Manag Res 7: 51-63, 2015.

5. Weis SM and Cheresh DA: Tumor angiogenesis: Molecular pathways and therapeutic targets. Nat Med 17: 1359-1370, 2011.

6. Redlitz A, Daum G and Sage EH: Angiostatin diminishes activation of the mitogen-activated protein kinases ERK-1 and ERK-2 in human dermal microvascular endothelial cells. J Vasc Res 36: 28-34, 1999.

7. O'Reilly MS, Holmgren L, Shing Y, Chen C, Rosenthal RA, Moses M, Lane WS, Cao Y, Sage EH and Folkman J: Angiostatin: A novel angiogenesis inhibitor that mediates the suppression of metastases by a Lewis lung carcinoma. Cell 79: 315-328, 1994.

8. Shichinohe T, Bochner BH, Mizutani K, Nishida M, Hegerich-Gilliam S, Naldini L and Kasahara N: Development of lentiviral vectors for antiangiogenic gene delivery. Cancer Gene Ther 8: 879-889, 2001.

9. O'Reilly MS, Boehm T, Shing Y, Fukai N, Vasios G, Lane WS, Flynn E, Birkhead JR, Olsen BR and Folkman J: Endostatin: An endogenous inhibitor of angiogenesis and tumor growth. Cell 88: 277-285, 1997.

10. Kuo CJ, Farnebo F, Yu EY, Christofferson R, Swearingen RA, Carter R, von Recum HA, Yuan J, Kamihara J, Flynn E, et al: Comparative evaluation of the antitumor activity of antiangiogenic proteins delivered by gene transfer. Proc Natl Acad Sci USA 98: 4605-4610, 2001.

11. Szentirmai O, Baker CH, Bullain SS, Lin N, Takahashi M, Folkman J, Mulligan RC and Carter BS: Successful inhibition of intracranial human glioblastoma multiforme xenograft growth via systemic adenoviral delivery of soluble endostatin and soluble vascular endothelial growth factor receptor-2: Laboratory investigation. J Neurosurg 108: 979-988, 2008.

12. Wu Y, Li ZY, Zhao X, Kan B and Wei YQ: Inhibition of ovarian tumor growth by gene therapy with recombinant soluble vascular endothelial growth factor receptor 2 . Hum Gene Ther 17: 941-948, 2006 . 
13. Zucali PA, Ceresoli GL, De Vincenzo F, Simonelli M, Lorenzi E, Gianoncelli L and Santoro A: Advances in the biology of malignant pleural mesothelioma. Cancer Treat Rev 37: 543-558, 2011.

14. Remon J, Lianes P, Martínez S, Velasco M, Querol R and Zanui M: Malignant mesothelioma: New insights into a rare disease. Cancer Treat Rev 39: 584-591, 2013.

15. Strizzi L, Catalano A, Vianale G, Orecchia S, Casalini A, Tassi G, Puntoni R, Mutti L and Procopio A: Vascular endothelial growth factor is an autocrine growth factor in human malignant mesothelioma. J Pathol 193: 468-475, 2001.

16. Kumar-Singh S, Weyler J, Martin MJ, Vermeulen PB and Van Marck E: Angiogenic cytokines in mesothelioma: A study of VEGF, FGF-1 and -2, and TGF beta expression. J Pathol 189: $72-78,1999$.

17. Yasumitsu A, Tabata C, Tabata R, Hirayama N, Murakami A, Yamada S, Terada T, Iida S, Tamura K, Fukuoka K, et al: Clinical significance of serum vascular endothelial growth factor in malignant pleural mesothelioma. J Thorac Oncol 5: 479-483, 2010.

18. Kourlas $\mathrm{H}$ and Abrams P: Ranibizumab for the treatment of neovascular age-related macular degeneration: A review. Clin Ther 29: 1850-1861, 2007.

19. Jenab-Wolcott $\mathbf{J}$ and Giantonio BJ: Bevacizumab: Current indications and future development for management of solid tumors. Expert Opin Biol Ther 9: 507-517, 2009.

20. Langer C and Soria JC: The role of anti-epidermal growth factor receptor and anti-vascular endothelial growth factor therapies in the treatment of non-small-cell lung cancer. Clin Lung Cancer 11: $82-90,2010$.

21. Ceresoli GL, Zucali PA, Mencoboni M, Botta M, Grossi F, Cortinovis D, Zilembo N, Ripa C, Tiseo M, Favaretto AG, et al: Phase II study of pemetrexed and carboplatin plus bevacizumab as first-line therapy in malignant pleural mesothelioma. $\mathrm{Br} J$ Cancer 109: 552-558, 2013

22. Dowell JE, Dunphy FR, Taub RN, Gerber DE, Ngov L, Yan J, Xie Y and Kindler HL: A multicenter phase II study of cisplatin, pemetrexed, and bevacizumab in patients with advanced malignant mesothelioma. Lung Cancer 77: 567-571, 2012.

23. Kubo S and Mitani K: A new hybrid system capable of efficient lentiviral vector production and stable gene transfer mediated by a single helper-dependent adenoviral vector. J Virol 77: 2964-2971, 2003.

24. Kubo S, Seleme MC, Soifer HS, Perez JL, Moran JV, Kazazian $\mathrm{HH} \mathrm{Jr}$ and Kasahara N: L1 retrotransposition in nondividing and primary human somatic cells. Proc Natl Acad Sci USA 103: 8036-8041, 2006.
25. Takahashi S: Vascular endothelial growth factor (VEGF), VEGF receptors and their inhibitors for antiangiogenic tumor therapy. Biol Pharm Bull 34: 1785-1788, 2011.

26. Bougatef F, Quemener C, Kellouche S, Naïmi B, Podgorniak MP Millot G, Gabison EE, Calvo F, Dosquet C, Lebbé C, et al: EMMPRIN promotes angiogenesis through hypoxia-inducible factor-2alpha-mediated regulation of soluble VEGF isoforms and their receptor VEGFR-2. Blood 114: 5547-5556, 2009.

27. Lee S, Chen TT, Barber CL, Jordan MC, Murdock J, Desai S, Ferrara N, Nagy A, Roos KP and Iruela-Arispe ML: Autocrine VEGF signaling is required for vascular homeostasis. Cell 130: 691-703, 2007.

28. Robinson CJ and Stringer SE: The splice variants of vascular endothelial growth factor (VEGF) and their receptors. J Cell Sci 114: 853-865, 2001.

29. Li Q, Yano S, Ogino H, Wang W, Uehara H, Nishioka Y and Sone S: The therapeutic efficacy of anti vascular endothelial growth factor antibody, bevacizumab, and pemetrexed against orthotopically implanted human pleural mesothelioma cells in severe combined immunodeficient mice. Clin Cancer Res 13 5918-5925, 2007.

30. Kawasaki Y, Tamamoto A, Takagi-Kimura M, Maeyama Y, Yamaoka N, Terada N, Okamura H, Kasahara N and Kubo S: Replication-competent retrovirus vector-mediated prodrug activator gene therapy in experimental models of human malignant mesothelioma. Cancer Gene Ther 18: 571-578, 2011.

31. Kubo S, Takagi-Kimura M, Logg CR and Kasahara N: Highly efficient tumor transduction and antitumor efficacy in experimental human malignant mesothelioma using replicating gibbon ape leukemia virus. Cancer Gene Ther 20: 671-677, 2013.

32. Kubo S, Kawasaki Y, Yamaoka N, Tagawa M, Kasahara N, Terada $\mathrm{N}$ and Okamura $\mathrm{H}$ : Complete regression of human malignant mesothelioma xenografts following local injection of midkine promoter-driven oncolytic adenovirus. J Gene Med 12: 681-692, 2010

33. Takagi-Kimura M, Yamano T, Tagawa M and Kubo S: Oncolytic virotherapy for osteosarcoma using midkine promoter-regulated adenoviruses. Cancer Gene Ther 21: 126-132, 2014.

34. Takagi-Kimura M, Yamano T, Tamamoto A, Okamura N Okamura H, Hashimoto-Tamaoki T, Tagawa M, Kasahara N and Kubo S: Enhanced antitumor efficacy of fiber-modified, midkine promoter-regulated oncolytic adenovirus in human malignant mesothelioma. Cancer Sci 104: 1433-1439, 2013. 